\title{
Long non-coding RNA FER1L4 inhibits cell proliferation and metastasis through regulation of the PI3K/AKT signaling pathway in lung cancer cells
}

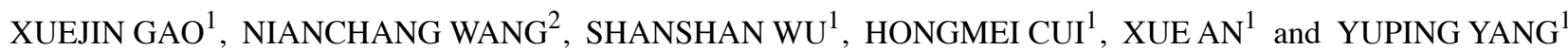 \\ ${ }^{1}$ Department of Respiratory and Critical Care Medicine, Xiqing Hospital, Tianjin 300380; ${ }^{2}$ Department of Oncology, \\ National Cancer Center/Cancer Hospital, Chinese Academy of Medical Sciences and Peking Union Medical College, \\ Beijing 100021, P.R. China
}

Received March 18, 2018; Accepted March 20, 2019

DOI: $10.3892 / \mathrm{mmr} .2019 .10219$

\begin{abstract}
Lung cancer is among the most common malignancies worldwide; however, the current understanding of its detailed mechanism remains limited. Long non-coding RNAs (lncRNAs) were previously identified to serve significant roles in tumorigenesis. The present study aimed to investigate the role of a novel lncRNA, Fer-1-like family member 4 (FER1L4), in lung tumorigenesis. In the present study, it was demonstrated that the expression level of FER1L4 was significantly decreased in clinical lung cancer tissues and in cultured lung cancer cells, as evidenced by reverse transcription-quantitative polymerase chain reaction analysis. Overexpression of FER1L4 in lung cancer cell lines A549 and 95D inhibited colony formation, cell proliferation and cell migration capacity, measured by colony formation assays, cell proliferation assays and Transwell assays, respectively. Overexpression of FER1L4 led to a reduction in the expression levels of phosphoinositide 3-kinase (PI3K)/protein kinase B (Akt) in A549 and 95D cells, whereas, activation of PI3K/Akt signaling using a small molecular inhibitor of phosphatase and tensin homolog, reversed the inhibitory effects of FER1L4 on cell proliferation and metastasis. All of these results suggested that the lncRNA FER1L4 suppressed cell proliferation and metastasis by inhibiting the PI3K/Akt signaling pathway in lung cancer.
\end{abstract}

\section{Introduction}

Lung cancer is one of the most common malignancies worldwide and it is characterized by uncontrolled cell growth in

Correspondence to: Dr Yuping Yang, Department of Respiratory and Critical Care Medicine, Xiqing Hospital, 403 Xiqing Road, Tianjin 300380, P.R. China

E-mail: yupingyang3344@163.com

Key words: Fer-1-like family member 4, proliferation, metastasis, phosphoinositide 3-kinase/protein kinase B signaling, lung cancer the lungs. The most common symptoms of lung cancer are coughing, weight loss, shortness of breath and chest pains (1). The number of novel diagnosed cases of lung cancer reached 1.8 million in 2012 and 1.6 million mortalities were reported in the same year, making lung cancer the most common cause of cancer-associated mortalities in males and the second most common cause in females, only secondary to breast cancer (2). The five-year survival rate of patients with lung cancer was $17.4 \%$ in the USA in 2008, whereas, the outcomes were worse in developing countries (3). Limited access to early diagnosis and treatment is the most important factor for the occurrence of clinical lung cancer worldwide (4). The majority of patients suffering from lung cancer additionally present local metastases due to the high metastatic potential of lung cancer cells (5).

Long non-coding RNAs (lncRNAs) are defined as RNAs with lengths $>200$ nucleotides that are not translated into proteins (6). IncRNA expression levels are 10-fold lower compared with mRNAs in various cell types, and the expression levels of lncRNA genes are more variable among cells, compared with protein-coding genes (7). Transcriptional profiles of various cell types using next generation sequencing demonstrated that tens of thousands of lncRNAs exist in mammals (8). Although accumulating evidence has demonstrated that the majority of these are likely to be functional, only a small proportion of IncRNAs are biologically relevant (9).

Previous studies demonstrated that numerous lncRNAs serve significant roles in tumorigenesis. IncRNA taurine upregulated gene 1 was downregulated and associated with cell apoptosis in human breast cancer (10). More specifically, in lung cancer, lncRNAs CDKN2B antisense RNA 1 and ENST457720 were reported to regulate cell proliferation in vivo and in vitro $(11,12)$. However, the detailed mechanisms underlying the regulatory roles of lncRNAs in human lung cancer require identification. Furthermore, at present, to the best of the authors' knowledge, lncRNAs have not been used in the diagnosis and treatment of lung cancer. Therefore, it is critical to identify novel lncRNAs involved in the progression of lung cancer.

In the present study, it was identified that a novel lncRNA, Fer-1-like family member 4 (FER1L4), serves roles in cell 
proliferation and metastasis of lung cancer. Furthermore, the mechanism underlying FER1L4 function in lung cancer was examined. These results provide novel insight of lung cancer progression, and may improve clinical diagnosis and treatment of lung cancer in the future.

\section{Materials and methods}

Human samples. The present study was approved by the Ethics Committee of Xiqing Hospital (Tianjin, China). In total, 100 patients with lung cancer (male:female ratio, 60:40; average age, 59 years old) from the Department of Respiration, Xiqing Hospital, were enrolled between January 2016 and December 2017. Informed written consent was obtained from all patients. No chemotherapies or radiotherapies were performed prior to surgery. During surgery, the lung cancer tissues and adjacent normal tissues were frozen in liquid nitrogen as soon as they were dissected from the patients, and stored until use for subsequent analysis.

Cell culture and transfection. The normal lung cell line BEAS-2B and lung cancer cell line SPC-A-1 were purchased from The American Type Culture Collection (Manassas, VA, USA). Other lung cancer cell lines A549, H1975, H-125 and 95D were obtained from The Cell Bank of Chinese Academy of Sciences (Shanghai, China). All cells were cultured in Dulbecco's modified Eagle's medium (DMEM) purchased from Gibco (Thermo Fisher Scientific, Inc., Waltham, MA, USA) supplied with $10 \%$ fetal bovine serum (FBS; Gibco; Thermo Fisher Scientific, Inc.) at $37^{\circ} \mathrm{C}$. A FER1L4 expression plasmid was constructed using a pcDNA 3.1 vector by Jie Li Biology (http://www.genebioseq.com/, Shanghai, China) with XhoI and HindIII restriction enzymes. A549, 95D and BEAS-2B cells were transfected with $2 \mu \mathrm{g} / \mathrm{ml}$ plasmid using Lipofectamine ${ }^{\circledR} 3000$ (Invitrogen; Thermo Fisher Scientific, Inc.) for $48 \mathrm{~h}$, according to the manufacturer's protocol. SF1670 (Selleck Chemicals, Shanghai, China), a specific inhibitor of phosphatase and tensin homolog (PTEN), was purchased to activate the phosphoinositide 3-kinase (PI3K)/protein kinase $\mathrm{B}$ (Akt) signaling pathway and diluted in dimethyl sulfoxide to a concentration of $50 \mathrm{mM}$. The working concentration of SF1670 was $50 \mu \mathrm{M}$ and the incubation time was $6 \mathrm{~h}$ at $37^{\circ} \mathrm{C}$.

RNA extraction and reverse transcription-quantitative polymerase chain reaction ( $R T-q P C R)$. Total RNA from the patient tissues and cultured cells were extracted using TRIzol $^{\circledR}$ (Thermo Fisher Scientific, Inc.) reagent, using a volume of $1 \mathrm{ml} /$ well in six-well plates. The RNA amount and quality were measured using Nanodrop 2000 (Thermo Fisher Scientific, Inc., Wilmington, DE, USA). A total of $500 \mathrm{ng}$ RNA was reverse transcribed into cDNA in a volume of $10 \mu \mathrm{l}$ using PrimeScript RT Reagent kit (Takara Biotechnology Co., Ltd., Dalian, China), at $37^{\circ} \mathrm{C}$ for $15 \mathrm{~min}$ and $85^{\circ} \mathrm{C}$ for 5 sec. RT-qPCR was performed in an ABI 7900 machine using a SYBR Green kit (Takara Biotechnology Co., Ltd.) according to the manufacturer's protocol. The thermocycling conditions were as follows: Initial denaturation at $95^{\circ} \mathrm{C}$ for $5 \mathrm{~min}$, followed by 45 repeats of a three-step cycling program consisting of $10 \mathrm{sec}$ at $95^{\circ} \mathrm{C}$ (denaturation), $10 \mathrm{sec}$ at $60^{\circ} \mathrm{C}$ (primer annealing) and $10 \mathrm{sec}$ at $72^{\circ} \mathrm{C}$ (elongation), and a final
Table I. Primers used in the present study.

\begin{tabular}{lll}
\hline Gene & Direction & \multicolumn{1}{c}{ Sequences } \\
\hline FER1L4 & Forward & 5'-AATGTGGGCTTCCAGGAAC-3' \\
& Reverse & 5'-CACCAGAAAGTTCCACGTC-3' \\
PI3K & Forward & 5'-GTCCTATTGTCGTGCATGTGG-3' \\
& Reverse & 5'-TGGGTTCTCCCAATTCAACC-3' \\
Akt & Forward & 5'- TTCTATGGCGCTGAGATTGTGT-3' \\
& Reverse & 5'-GCCGTAGTCATTGTCCTCCAG-3' \\
GAPDH & Forward & 5'-GGTCGGAGTCAACGGATTTG-3' \\
& Reverse & 5'-GGAAGATGGTGATGGGATTTC-3'
\end{tabular}

FER1L4, Fer-1-like family member 4; PI3K, phosphoinositide 3-kinase; Akt, protein kinase B.

extension step for $10 \mathrm{~min}$ at $72^{\circ} \mathrm{C}$. Primers were synthesized by Jie Li Biology. and the sequences are listed in Table I. GAPDH was included as an internal control and $2^{-\Delta \Delta \mathrm{Cq}}$ method was used for statistical analysis (13).

Colony formation assay. A total of $1 \times 10^{5}$ cells were seeded into 12-well plates $24 \mathrm{~h}$ prior to the transfection with the FER1L4 expression plasmid. A total of 500 A549 and 95D cells were seeded into separate six-well plates. The plates were incubated at $37^{\circ} \mathrm{C}$ in an incubator $\left(5 \% \mathrm{CO}_{2}\right)$ for 2 weeks without changing the culture medium. Subsequently, the colonies were stained with crystal violet $(1 \%)$ at room temperature for $10 \mathrm{~min}$ and five random fields were imaged for each group with a Nikon light microscope (x200; Nikon Corporation, Tokyo, Japan). The number of cells was counted and statistical analyses were performed.

Cell proliferation assay. An MTT assay (Promega Corporation, Madison, WI, USA) was performed to examine the effects of FER1L4 on cell growth. A549 and 95D cells were transfected with FER1L4 expression plasmid and seeded into 96-well plates with an initial concentration of $5 \times 10^{3} /$ well. Each experimental group of cells was seeded in sextuplicate and the culture medium was replaced every other day. Cell proliferation was assessed at days 1,3 and 5, and DMSO was used to dilute formazan and stop the reaction. During the experiments, the cell proliferative rate was determined with a TECAN reader (Tecan Group, Ltd., Männedorf, Switzerland) with an absorbance of $490 \mathrm{~nm}$.

Transwell and Matrigel assays. For the cell migration assays, A549, 95D cells and BEAS-2B cells were transfected with the FER1L4-expressing plasmid for $48 \mathrm{~h}$ and collected by low-speed centrifugation $\left(1,000 \mathrm{x} \mathrm{g} ; 4^{\circ} \mathrm{C} ; 5 \mathrm{~min}\right)$ with serum-free DMEM. A total of $1 \times 10^{4}$ cells $(\sim 150 \mu \mathrm{l})$ were seeded into the upper chambers ( $8 \mu \mathrm{m}$ pore; Corning, Inc., Corning, NY, USA). The lower chambers were filled with $600 \mu \mathrm{l}$ medium containing $10 \%$ FBS. The plate was subsequently incubated at $37^{\circ} \mathrm{C}$. At $12 \mathrm{~h}$ post-seeding, the membrane was washed with PBS, fixed with precooled methanol (5 min, room temperature) and stained with crystal violet (1\%) for $5 \mathrm{~min}$ at room temperature. Cell migration was determined by counting the cells migrated through 
Table II. Association of FER1L4 with clinicopathological characteristics among 100 patients with lung cancer.

Expression of FER1L4

Clinicopathological characteristics

Number of patients

Low, $\mathrm{n}=57$

High, n=43

P-value

Age, years

$<40$

40-50

15

23

8

0.958

$>50$

62

7

Sex

13

36

0.683

Male

60

33

26

Female

40

24

Presenting symptoms

Painless lump

41

35

Painful lump

Atypical symptoms

24

Tumor size, $\mathrm{T}$

$\mathrm{T} 1, \leq 2 \mathrm{~cm}$

$$
17
$$

32

$\mathrm{T} 2,>2 \mathrm{~cm}$ but $<5 \mathrm{~cm}$

\section{8}

$\mathrm{T} 4$, any size with distant metastasis

23

Lymph node metastasis, $\mathrm{N}$

No

N1 or above

45

55

20

19

18

$<0.001^{\mathrm{a}}$

Distant metastasis, M

M0

42

M1

58

TNM stage

$\begin{array}{ll}\text { I/II } & 34 \\ \text { III/IV } & 66\end{array}$

$3 \quad 14$

$14 \quad 18$

$20 \quad 8$

$20 \quad 3$

$17 \quad 28$

$40 \quad 15$

$16 \quad 26$

$41 \quad 17$

$10 \quad 24$

$47 \quad 19$
III/IV

$66 \quad 47$

${ }^{\text {a }}<0.05$. TNM, tumor, node and metastasis; FER1L4, Fer-1-like family member 4.
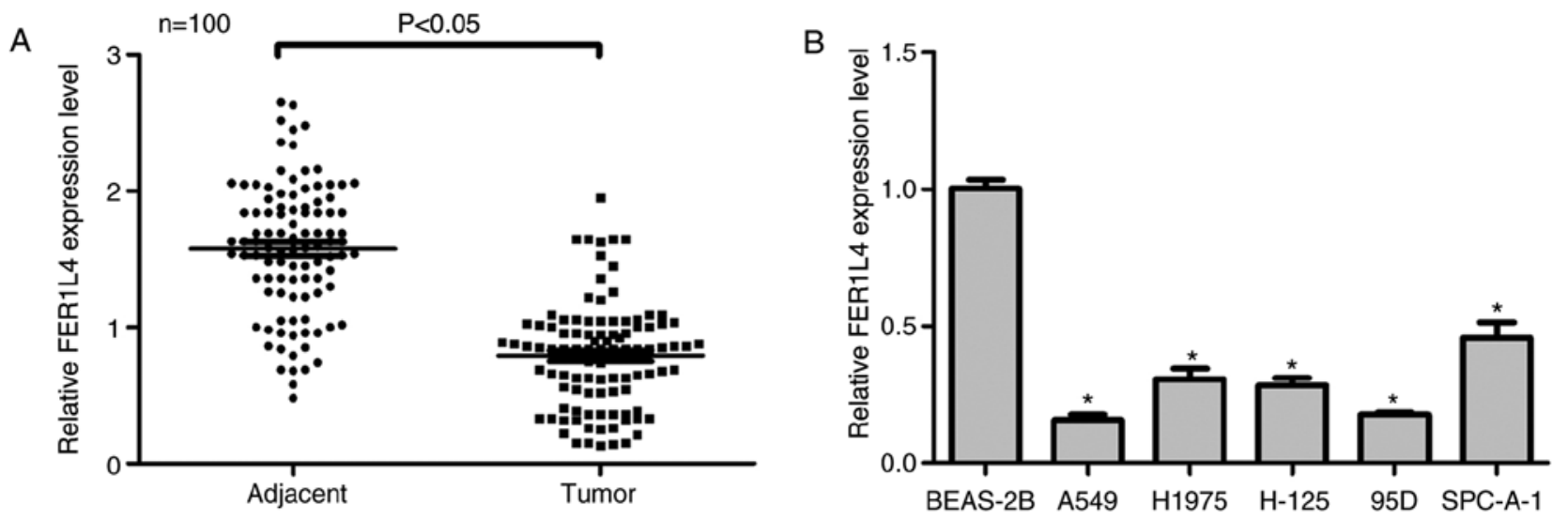

Figure 1. Expression level of FER1L4 is downregulated in lung cancer in vivo and in vitro. (A) RT-qPCR analysis was performed in 100 patients with lung cancer to examine the expression of FER1L4. (B) RT-qPCR analysis was performed in normal lung epithelial BEAS-2B cells and five lung cancer cell lines to examine the expression of FER1L4. ${ }^{*} \mathrm{P}<0.05$ vs. BEAS-2B. RT-qPCR, reverse transcription-quantitative polymerase chain reaction; FER1L4, Fer-1-like family member 4.

the membrane. In total, five random areas were imaged using a Nikon light microscope (Nikon Corporation; magnification, $\mathrm{x} 200)$. For cell invasion assays, the membrane was pre-coated with Matrigel (Corning, Inc.) for $6 \mathrm{~h}$ at $37^{\circ} \mathrm{C}$. 
A

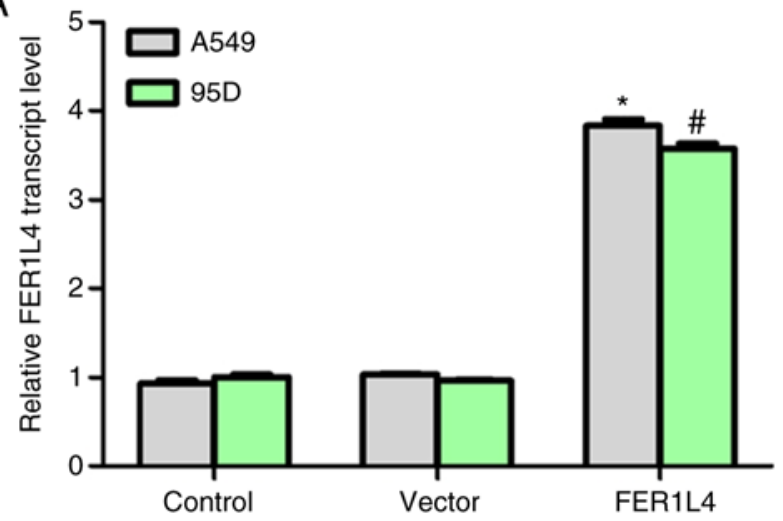

C

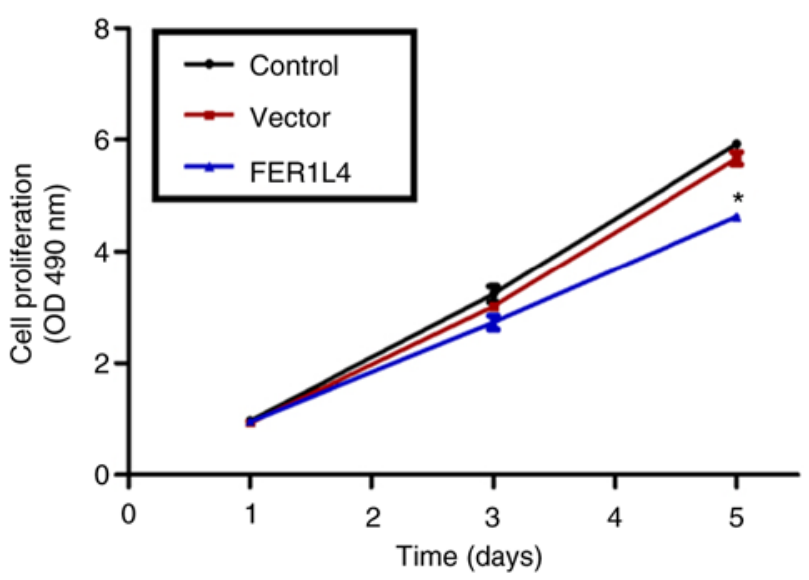

B 口4549

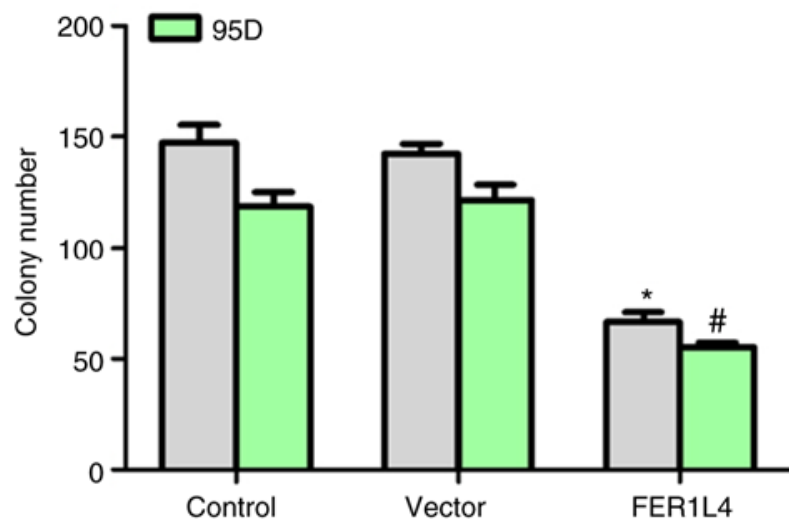

D

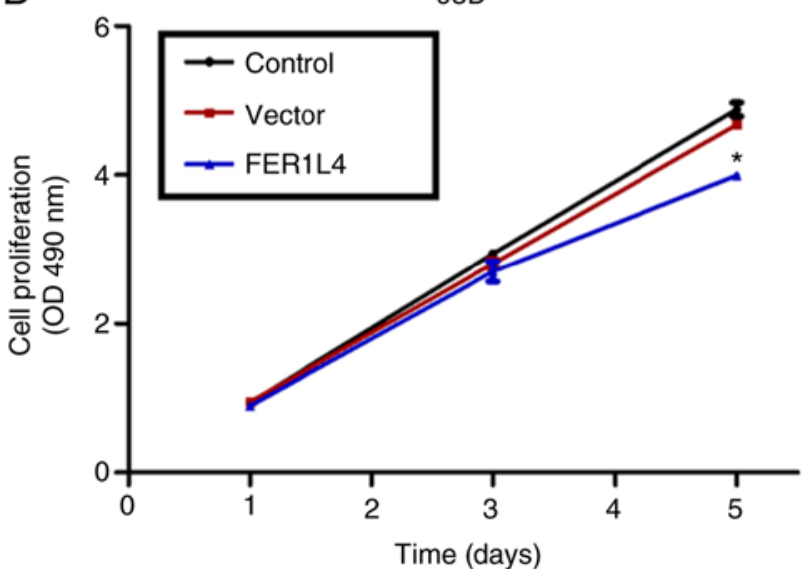

Figure 2. Overexpression of FER1L4 in A549 and 95D cells inhibits cell proliferation in vitro. (A) Reverse transcription-polymerase chain reaction analysis was performed to test the transfection efficiency. (B) A colony formation assay was performed following transfection of FER1L4 expression plasmid in A549 and $95 \mathrm{D}$ cells. " $\mathrm{P}<0.05$ vs. Control in A549 cells; ${ }^{*} \mathrm{P}<0.05$ vs. Control in $95 \mathrm{D}$ cells. (C) Cell proliferation was examined by an MTT assay in A549 cells at day 1, 3 and 5 after transfection of the FER1L4 plasmid. (D) Cell proliferation was examined by an MTT assay in 95D cells at day 1, 3 and 5 after transfection of the FER1L4 plasmid. "P<0.05 vs. respective Control. FER1L4, Fer-1-like family member 4; OD, optical density.

Western blot analysis. Western blotting analysis was performed with cell lysates. Total protein from cultured A549 and 95D cells transfected with or without the FER1L4 expression plasmid were extracted using radioimmunoprecipitation assay lysis buffer (Beyotime Institute of Biotechnology, Haimen, China). The protein amount was determined with a bicinchoninic acid kit (Thermo Fisher Scientific, Inc.). Subsequently, a total of $40 \mu \mathrm{g}$ protein was loaded onto a $10 \%$ SDS-PAGE gel and transferred to a polyvinylidene difluoride membrane (EMD Millipore, Billerica, MA, USA). Subsequent to blocking with 5\% skim milk for $1 \mathrm{~h}$ at room temperature, the membranes were incubated with primary antibodies diluted in $5 \%$ bovine serum albumin (Gibco; Thermo Fisher Scientific, Inc.) solution. The following primary antibodies were all purchased from Cell Signaling Technology, Inc. (Danvers, MA, USA): PI3K (cat. no. 4257; 1:1,000), Akt (cat. no. 9272; 1:1,000), phosphorylated (p-)Akt (cat. no. 9271; 1:1,000) and GAPDH (cat. no. 5174; 1:5,000). p-PI3K antibody was purchased from BioWorld Technology Co., Ltd. (cat. no. BS4605; 1:500; Nanjing, China). The membrane was incubated with primary antibodies at $4^{\circ} \mathrm{C}$ overnight, followed by incubation with secondary antibodies for $1 \mathrm{~h}$ at room temperature. The horseradish peroxidase-conjugated goat anti-rabbit secondary antibody was purchased from Santa Cruz Biotechnology, Inc. (cat. no. sc-2004; 1:5,000; Dallas, TX, USA). Immunoreactive bands were visualized using an enhanced chemiluminescent system (Thermo Fisher Scientific, Inc.).

Wound healing assays. A549 and 95D cells were transfected with FER1L4 expression plasmid for $48 \mathrm{~h}$ and were subsequently cultured in DMEM in a six-well culture plate at a density of $5 \times 10^{5}$ cells/well. When the confluence reached $95 \%$, the cells were washed and the culture medium was replaced with serum-free DMEM. A scratch was made through the single cell layer using a $10 \mu \mathrm{l}$ pipette tip and the cells were washed again with warmed PBS. After an incubation of $24 \mathrm{~h}$, images of the migrating cells were captured with a Nikon light microscope (magnification, $\mathrm{x} 200$ ).

Statistical analysis. All data are presented as the mean \pm standard deviation. GraphPad Prism 5.0 (GraphPad Software, Inc., La Jolla, CA, USA) was used for analysis. Each experiment was repeated at least three times, unless otherwise stated. The two-tailed Student's t-test was used to compare means between two groups, whereas, one-way analysis of variance was used for comparisons among multiple groups (more than two groups), 


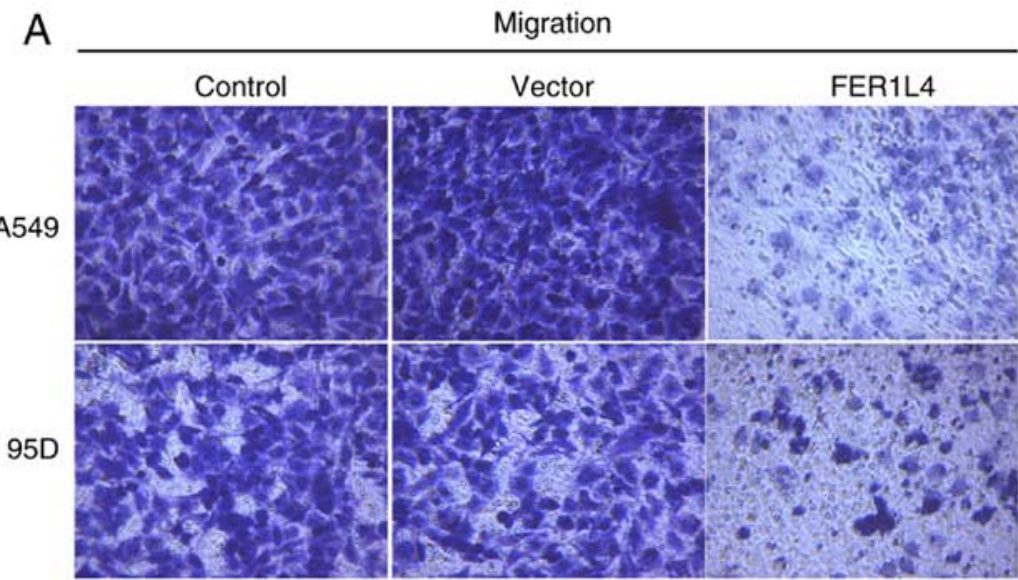

B

Invasion

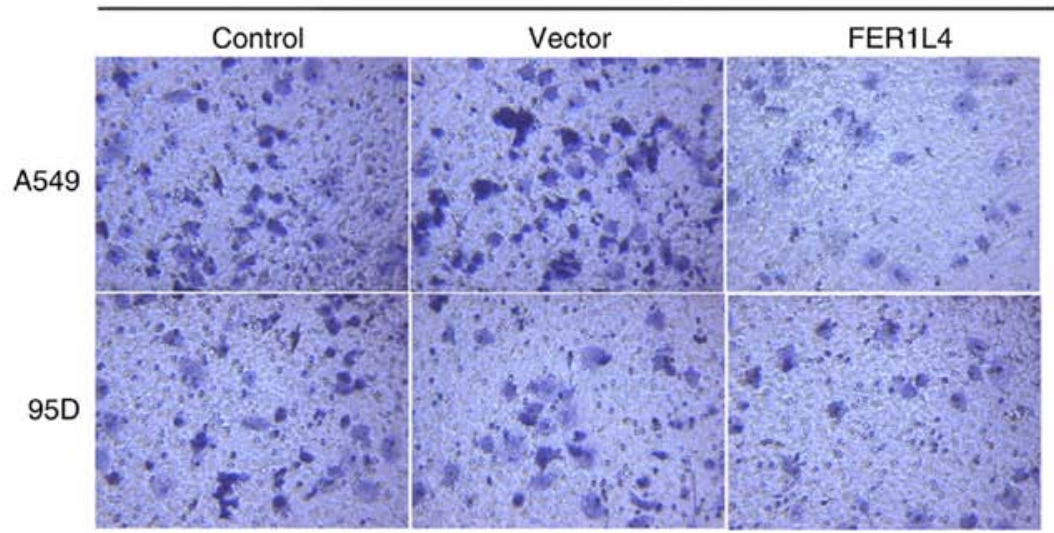

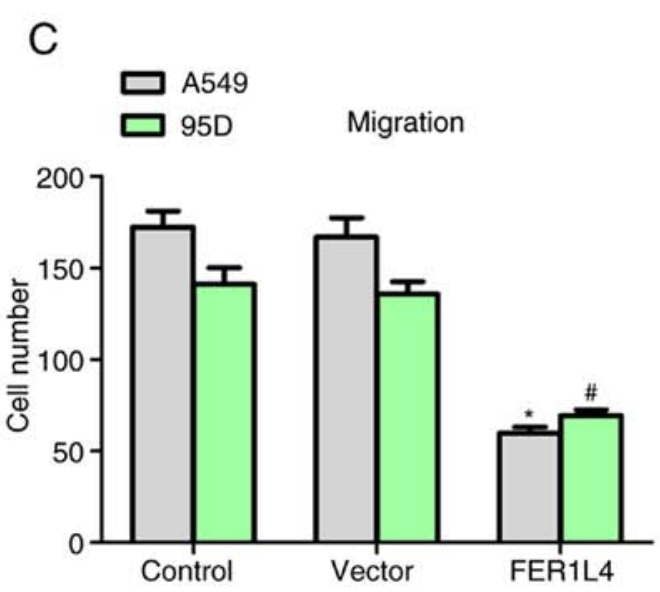

D

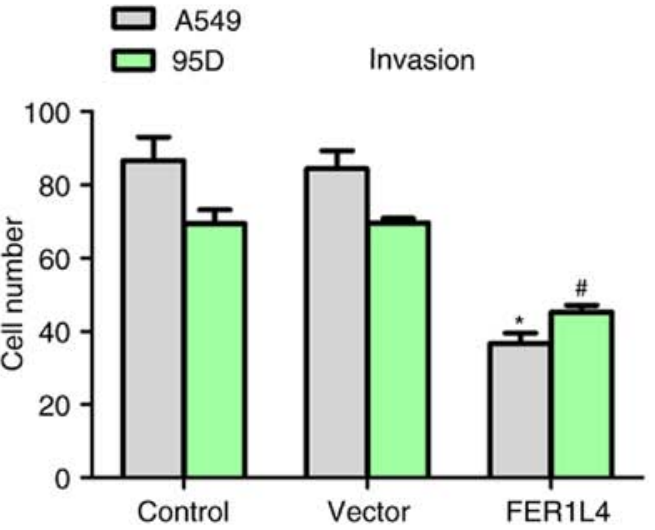

Figure 3. Overexpression of FER1L4 in A549 and 95D cells suppresses cell metastasis in vitro. (A) Representative images of the transwell cell migration assay. (B) Representative images of the Matrigel cell invasion assay. Magnification, x200. (C) Quantification of cell migration in A549 and 95D cells following transfection of the FER1L4 expression plasmid. (D) Quantification of cell invasion in A549 and 95D cells following transfection of the FER1L4 expression plasmid. " $\mathrm{P}<0.05$ vs. Control in A549 cells; ${ }^{\#} \mathrm{P}<0.05$ vs. Control in 95D cells. FER1L4, Fer-1-like family member 4.

followed by Fisher's least significant difference post-hoc test. $\chi^{2}$ or Fisher's exact test were used to compare proportion differences of categorical variables. $\mathrm{P}<0.05$ was considered to indicate a statistically significant difference.

\section{Results}

mRNA expression level of FERIL4 is downregulated in lung cancer in vivo and in vitro. To examine the role of FER1L4, its expression in human lung cancer was assessed. In total, 100 patients with lung cancer were included in the present study and it was demonstrated by RT-qPCR that the expression level of FER1L4 was significantly downregulated in patients with lung cancer (Fig. 1A). The clinical features of the 100 patients with lung cancer and the relative expression of FER1L4 were additionally analyzed (Table II). It was demonstrated that the expression level of FER1L4 was not associated with age, sex or presenting symptoms of the 
A
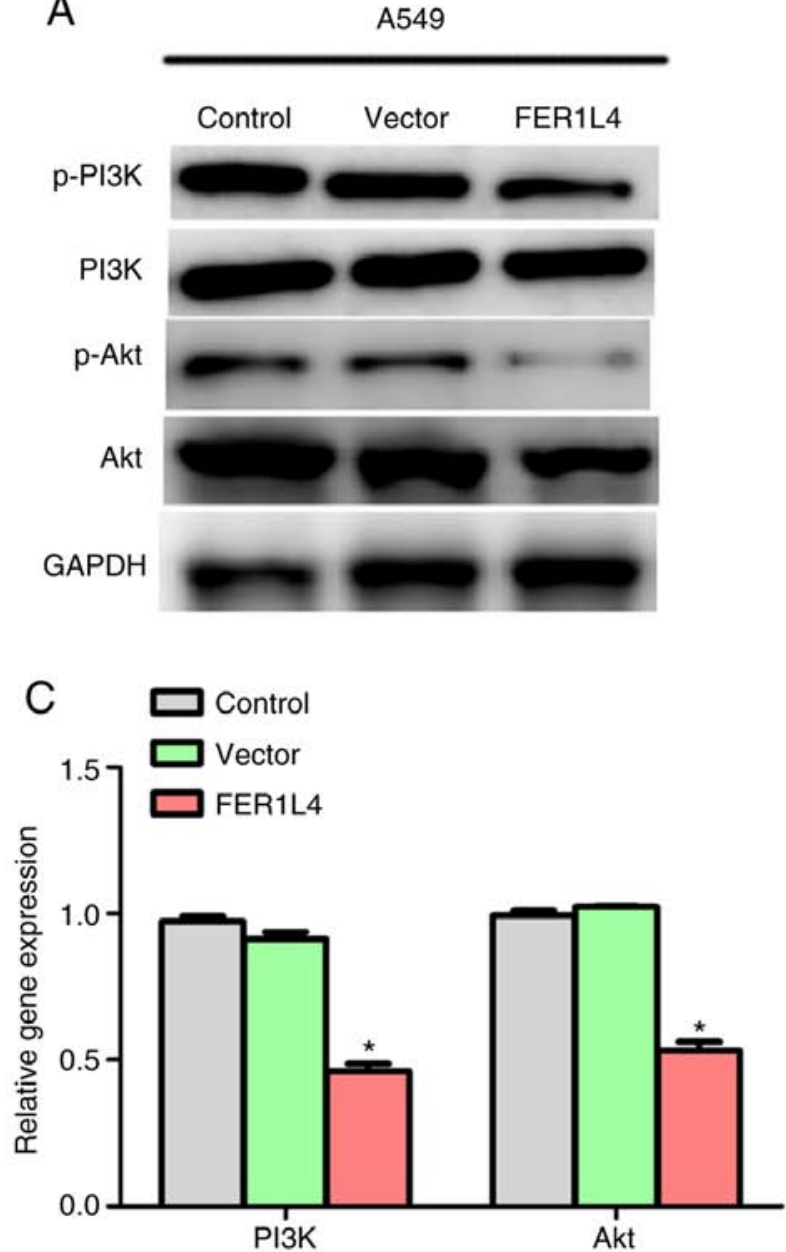

B
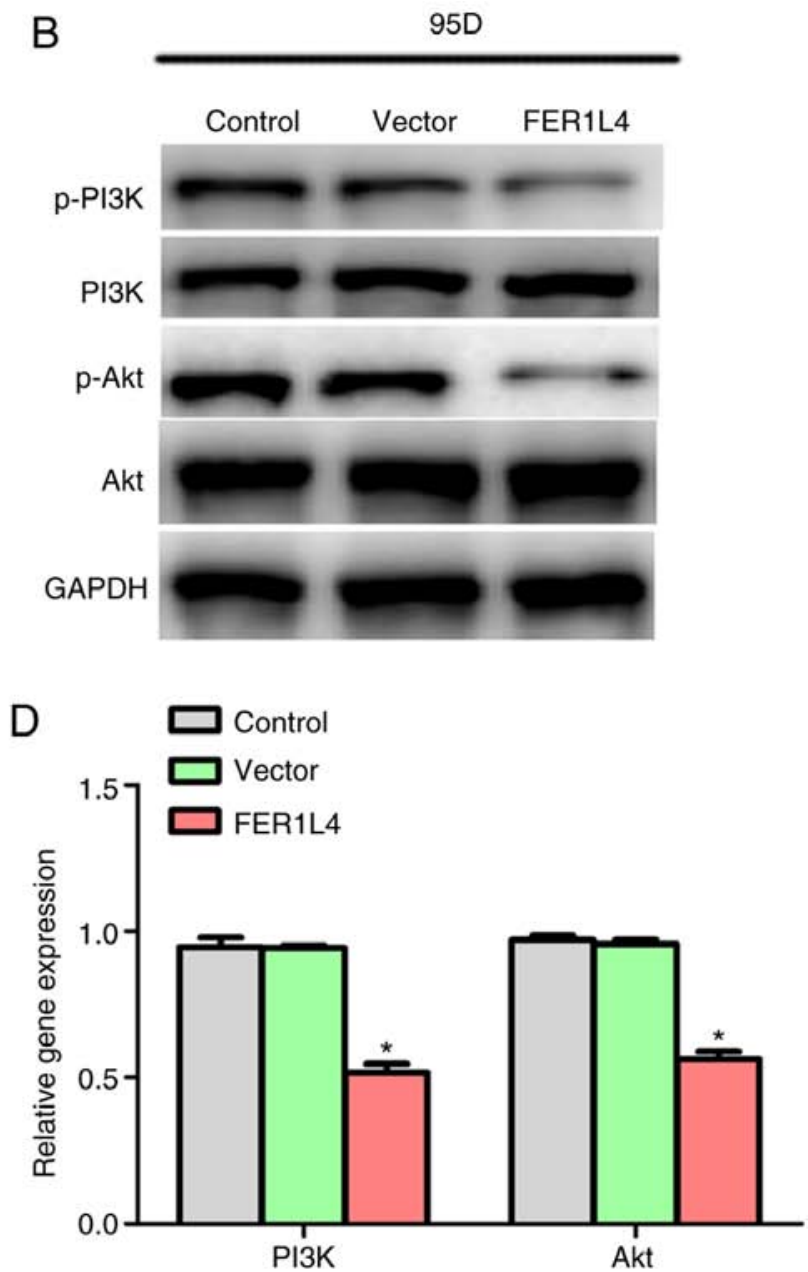

Figure 4. Upregulation of FER1L4 suppresses the PI3K/Akt signaling pathway in human lung cancer. (A) Western blot analysis depicting the protein expression levels of PI3K and Akt, in addition to their phosphorylation levels in A549 cells transfected with the FER1L4 expression plasmid. (B) Western blot analysis depicting the protein expression levels of PI3K and Akt, in addition to their phosphorylation levels in 95D cells transfected with the FER1L4 expression plasmid. GAPDH was used as a control. (C) RT-qPCR analysis was performed to measure the mRNA expression levels of PI3K and Akt in A549 cells transfected with FER1L4 expression plasmid. (D) RT-qPCR analysis was performed to measure the mRNA expression levels of PI3K and Akt in 95D cells transfected with FER1L4 expression plasmid. "P<0.05 vs. respective Control. RT-qPCR, reverse transcription-quantitative polymerase chain reaction; FER1L4, Fer-1-like family member 4; PI3K, phosphoinositide 3-kinase; Akt, protein kinase B; p-, phosphorylated.

patients; however, FER1L4 expression level was associated with tumor size, lymph node metastasis, distant metastasis and tumor, node and metastasis (TNM) staging. The normal lung epithelia cell line BEAS-2B and five lung cancer cell lines were assessed for FER1L4 gene expression. In all of the five cancer cell lines, the expression levels of FER1L4 were consistently downregulated compared with the BEAS-2B cell line. In particular, A549 and 95D cells, the two cell lines with the highest migration potential (4), exhibited the lowest expression levels of FER1L4 (Fig. 1B). The present results demonstrated that the expression level of FER1L4 is downregulated in human lung cancer in vivo and in vitro, and suggested a potential association between FER1L4 expression levels and tumor malignancy.

Overexpression of FER1L4 in A549 and 95D cells inhibits cell proliferation in vitro. An expression plasmid of FER1L4 was constructed and transfected into A549 and 95D cells and a significant increase in the expression level of FER1L4 was observed following transfection (Fig. 2A), up to 3.5-fold compared with the control groups. The effects of FER1L4 were examined by performing colony formation assays and cell proliferation assays. In total, $\sim 150$ and 125 colonies were detected in the control A549 and 95D cells, respectively. However, only 70 and 60 colonies were observed for A549 and 95D cells following overexpression of FER1L4 (Fig. 2B). However, a similar colony number was counted in the control cell line BEAS-2B with or without overexpression of FER1L4 (data not shown). In addition, there was no marked difference in the proliferation rate among the three groups (control, vector and FER1L4 overexpression) in the first 3 days, whereas, overexpression of FER1L4 led to a reduction in the cell proliferation rate by 30 and $25 \%$ in A549 and 95D cells at day 5, respectively (Fig. $2 \mathrm{C}$ and D). These data demonstrated that the IncRNA FER1L4 inhibited cell proliferation in the human lung cancer cell lines A549 and 95D.

Overexpression of FER1L4 in A549 and 95D cells inhibits cell metastasis in vitro. Uncontrolled cell proliferation and metastasis are characteristic of malignant tumors (14). Hence, 
A
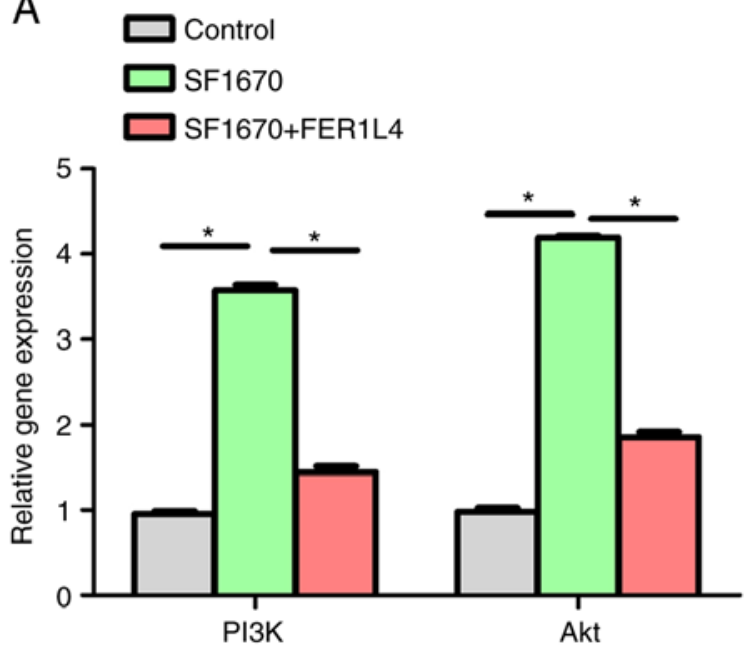

C

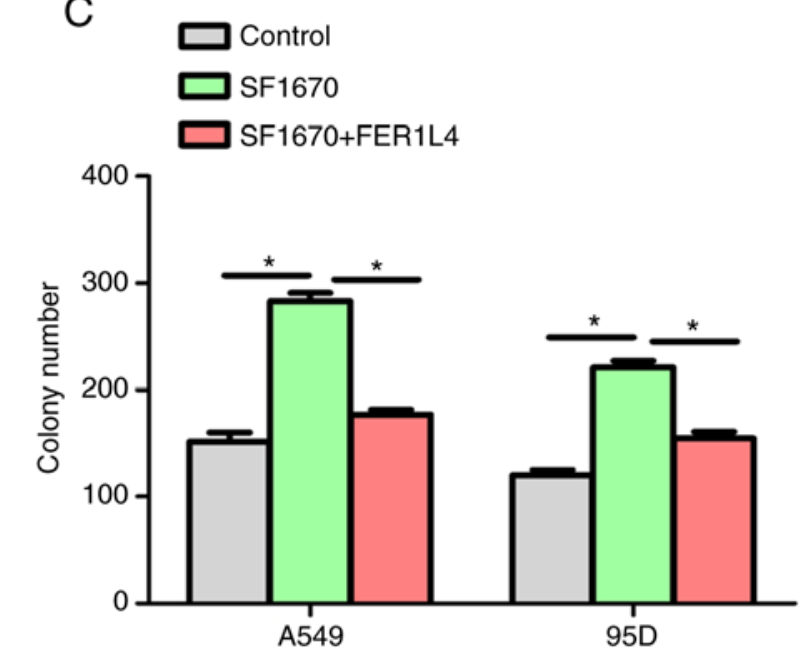

E 95D

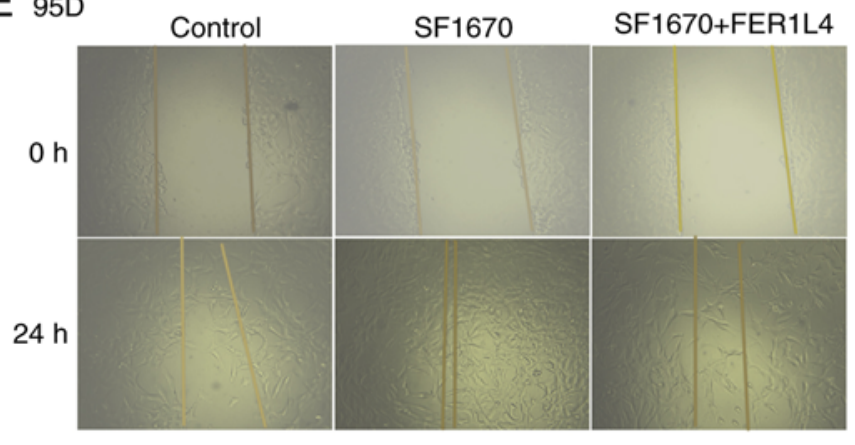

$\mathrm{B}$
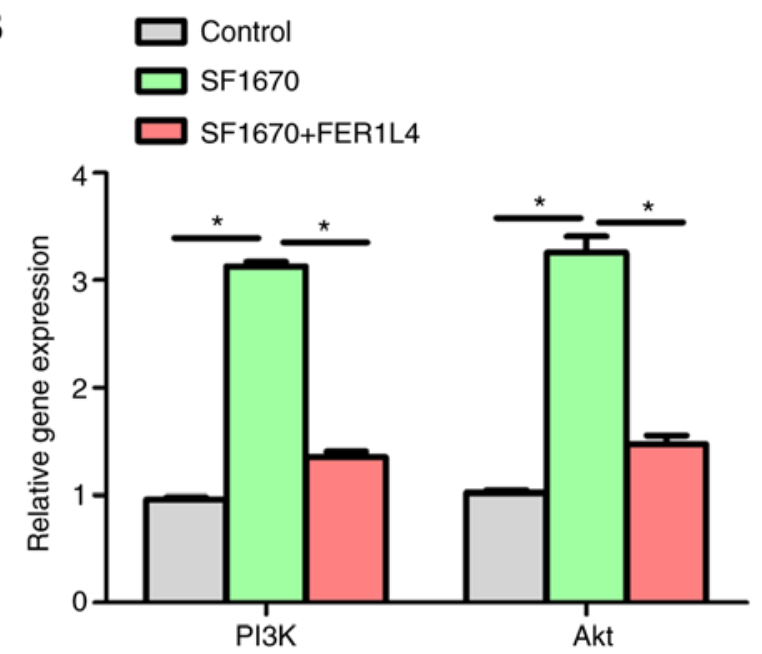

$\mathrm{D}$
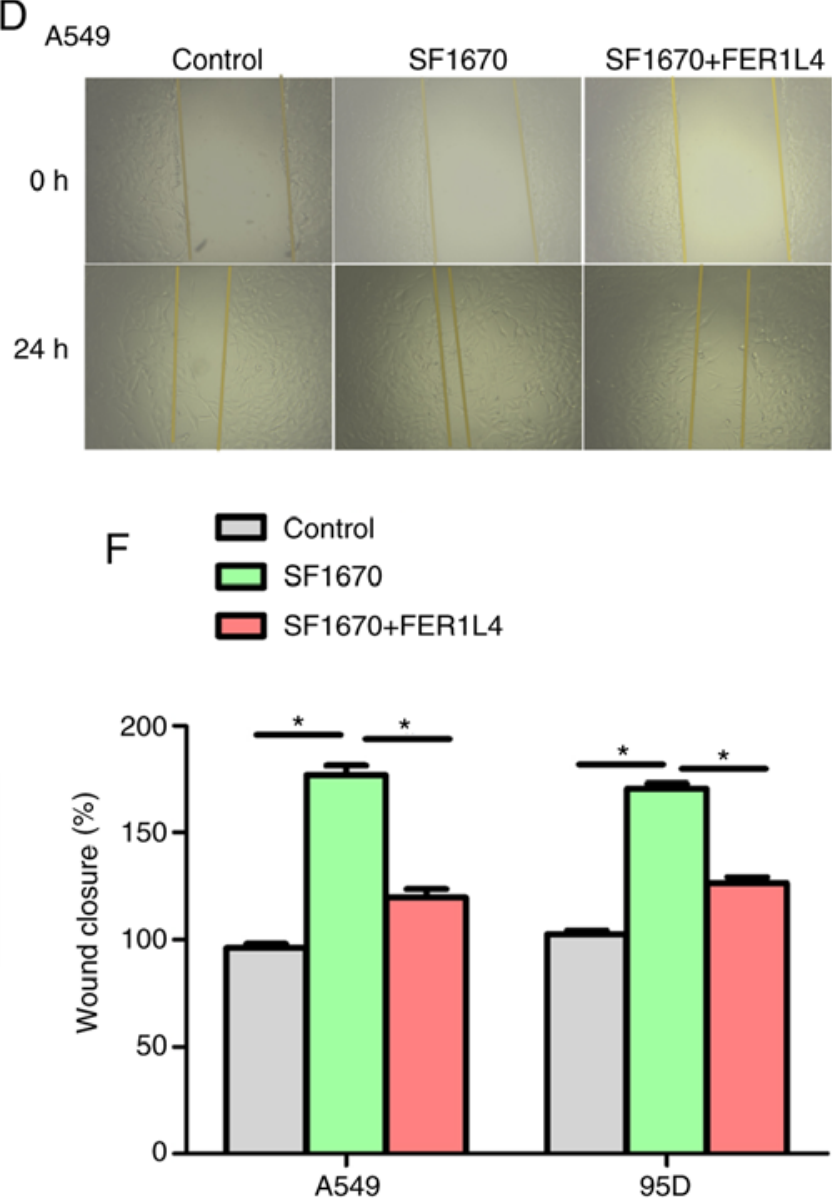

Figure 5. FER1L4 inhibits cell proliferation and metastasis via downregulation of the PI3K/Akt signaling pathway in human lung cancer. (A) mRNA expression levels of PI3K and Akt were examined in A549 cells transfected with FER1L4 expression plasmid in the presence or absence of the PTEN inhibitor SF1670. (B) mRNA expression levels of PI3K and Akt were examined in 95D cells transfected with FER1L4 expression plasmid in the presence or absence of the PTEN inhibitor SF1670. (C) A colony formation assay was performed for A549 cells transfected with FER1L4 expression plasmid in the presence or absence of PTEN inhibitor SF1670. (D) Representative images of the wound healing assay for A549 cells. (E) Representative images of the wound healing assay for 95D cells. Magnification, x200. (F) Quantification of the wound healing assay for A549 and 95D cells transfected with the FER1L4 expression plasmid in the presence or absence of the PTEN inhibitor SF1670. "P<0.05. FER1L4, Fer-1-like family member 4; PI3K, phosphoinositide 3-kinase; Akt, protein kinase B; PTEN, phosphatase and tensin homolog.

the role of FER1L4 in cell metastasis was tested (Fig. 3). As depicted in Fig. 3A and C, 170 A549 cells and 145 95D cells migrated through the membrane in the control conditions, whereas, following transfection with the FER1L4 expression plasmid only 60 A549 cells and 65 95D cells were observed on the lower surface of the chamber. In the invasion assays, cell invasion was additionally inhibited by $~ 50 \%$ in A549 and 95D cells when FER1L4 was overexpressed (Fig. 3B and D). Notably, the wound closure rate of BEAS-2B cells was approximately the same in the presence or absence of the FER1L4 
overexpression plasmid (data not shown). Collectively, the present results demonstrated that overexpression of FER1L4 in human lung cancer inhibited cell metastasis in A549 and 95D cells.

Upregulation of FERIL4 inhibits the PI3K/Akt signaling pathway in lung cancer cells. To examine the regulatory mechanism of FER1L4 in human lung cancer, the downstream signaling of FER1L4 in A549 and 95D cells was investigated. Western blot analysis was performed and it was identified that the protein expression levels of p-PI3K and p-AKT were decreased when FER1L4 was overexpressed in A549 and 95D cells (Fig. 4A and B). Furthermore, the expression levels of PI3K and Akt were examined. As depicted in Fig. 4C and D, when A549 and 95D cells were transfected with the FER1L4 expression plasmid, the expression levels of PI3K and Akt were downregulated by $\sim 50 \%$. These results suggested that upregulation of FER1L4 suppressed the PI3K/Akt signaling pathway in lung cancer.

FER1L4 inhibits cell proliferation and metastasis by downregulating the PI3K/Akt signaling pathway in lung cancer cells. To test whether FER1L4 regulates cell proliferation and metastasis through the PI3K/Akt signaling pathway, a specific inhibitor of PTEN was used, SF1670. Treatment with SF1670 led to an upregulation of the expression levels of PI3K and Akt by $>3$-fold in A549 cells, whereas, overexpression of FER1L4 restored the expressions of PI3K and Akt to relative normal levels (Fig. 5A). Similar results were obtained in 95D cells (Fig. 5B). Subsequently, functional experiments were performed in A549 and 95D cells. As presented in Fig. 5C, treatment with SF1670 increased the colony numbers in A549 and 95D cells, and colony formation in the two cell lines was decreased by FER1L4 overexpression, thus indicating that FER1L4 inhibited cell proliferation via regulation of the PI3K/Akt signaling pathway. Furthermore, a wound healing assay was performed and it was identified that treatment with SF1670 increased cell migration, whereas, overexpression of FER1L4 limited cell migration in A549 and 95D cells (Fig. 5D-F), further suggesting that FER1L4 inhibited cell metastasis via suppression of PI3K/Akt signaling pathway. Collectively, the present data demonstrated that FER1L4 inhibited cell proliferation and metastasis via downregulation of the PI3K/Akt signaling pathway in lung cancer cells.

\section{Discussion}

Lung cancer is among the most common malignancies in males and females worldwide (14). Although substantial efforts have been made in the past decades, the five-year survival rate of patients with lung cancer is still low in developed and developing countries due to a late diagnosis (15). Therefore, it is crucial to identify novel therapeutic targets against lung cancer.

FER1L4 is a novel lncRNA, which serves significant roles in various diseases. In 2013, it was identified that FER1L4 is downregulated in human gastric cancer (16) and later, in 2014, its role was described by Liu et al (17) in gastric cancer. The expression levels of FER1L4 were subsequently investigated in colon cancer (18), goat ovarian cancer (19), hepatocellular carcinoma (20) and glioma (21). Despite the characterization of its expression profile, the functional roles of FER1L4 and its mechanism of action in solid tumors remains unclear (17). In particular, its expression profile and biological roles in human lung cancer have not yet been identified. In the present study, it was demonstrated that FER1L4 is downregulated in lung cancer in vivo and in vitro. Its expression levels were associated with lung cancer clinicopathological parameters, including TNM staging, lymph node metastasis, distant metastasis and tumor size. Overexpression of FER1L4 inhibited cell proliferation and metastasis via regulation of the PI3K/Akt signaling pathway. Collectively, the present results suggested that FER1L4 may serve as a potential therapeutic target for lung cancer.

Numerous signaling pathways are involved in tumorigenesis, and the PI3K/Akt pathway is an important one (22). The $\mathrm{PI} 3 \mathrm{~K} /$ Akt signaling is aberrantly activated in human malignancies and is associated with tumor metastasis and drug resistance (23). The PI3K/Akt signaling pathway regulates the expression of snail family transcriptional repressor 1 and thus epithelial-mesenchymal transition, making the PI3K/Akt pathway a crucial target in clinical research (24). A principal antagonist of PI3K/Akt signaling is PTEN, a tumor suppressor that is frequently affected in a number of types of cancer (25). In the present study, it was identified that the lncRNA FER1L4 regulated the activity of the PI3K/Akt signaling pathway in human lung cancer. SF1670, a specific inhibitor of PTEN, was used as an activator of PI3K/Akt signaling and the present data suggested that activation of the PI3K/Akt signaling pathway rescued the inhibitory effects of FER1L4 on cell proliferation and metastasis in A549 and 95D cells, suggesting that FER1L4 serves significant roles in human lung cancer via the repression of the PI3K/Akt signaling pathway.

Collectively, the present study demonstrated that FER1L4 may serve as a tumor suppressor in lung cancer. Identification of specific activators of this lncRNA and identifying the detailed mechanisms underlying its regulatory effects on cell proliferation and metastasis may guide the development of novel drugs to treat lung cancer.

\section{Acknowledgements}

Not applicable.

\section{Funding}

No funding was received.

\section{Availability of data and materials}

The datasets used and/or analyzed during the current study are available from the corresponding author on reasonable request.

\section{Authors' contributions}

XG and NW performed the experiments. SW, HC and XA contributed to the data analysis and discussion. YY designed the study and prepared the manuscript. 


\section{Ethics approval and consent to participate}

The present study was approved by the Ethics Committee of Xiqing Hospital (Tianjin, China). Informed written consent was obtained from all patients.

\section{Patient consent for publication}

Not applicable.

\section{Competing interests}

The authors declare that they have no competing interests.

\section{References}

1. Hogan DB: Did Osler suffer from 'paranoia antitherapeuticum baltimorensis'? A comparative content analysis of The Principles and Practice of Medicine and Harrison's Principles of Internal Medicine, 11th edition. CMAJ 161: 842-845, 1999.

2. McGuire S: World Cancer Report 2014. Geneva, Switzerland: World Health Organization, International Agency for Research on Cancer, WHO Press, 2015. Adv Nutr 7: 418-419, 2016.

3. Majumder S, Chatterjee S, Pal S, Biswas J, Efferth T and Choudhuri SK: The role of copper in drug-resistant murine and human tumors. Biometals 22: 377-384, 2009

4. Peng G, Tisch U, Adams O, Hakim M, Shehada N, Broza YY, Billan S, Abdah-Bortnyak R, Kuten A and Haick H: Diagnosing lung cancer in exhaled breath using gold nanoparticles. Nat Nanotechnol 4: 669-673, 2009.

5. DeSantis CE, Siegel RL, Sauer AG, Miller KD, Fedewa SA Alcaraz KI and Jemal A: Cancer statistics for African Americans, 2016: Progress and opportunities in reducing racial disparities. CA Cancer J Clin 66: 290-308, 2016.

6. Brosnan CA and Voinnet O: The long and the short of noncoding RNAs. Curr Opin Cell Biol 21: 416-425, 2009.

7. Yunusov D, Anderson L, DaSilva LF, Wysocka J, Ezashi T, Roberts RM and Verjovski-Almeida S: Corrigendum: HIPSTR and thousands of IncRNAs are heterogeneously expressed in human embryos, primordial germ cells and stable cell lines. Sci Rep 6: 32753, 2016.

8. Li Y, Chen H, Pan T, Jiang C, Zhao Z, Wang Z, Zhang J, Xu J and Li X: LncRNA ontology: Inferring lncRNA functions based on chromatin states and expression patterns. Oncotarget 6: 39793-39805; 2015.

9. Mercer TR, Dinger ME and Mattick JS: Long non-coding RNAs: Insights into functions. Nat Rev Genet 10: 155-159, 2009.

10. Mourtada-Maarabouni M, Pickard MR, Hedge VL, Farzaneh F and Williams GT: GAS5, a non-protein-coding RNA, controls apoptosis and is downregulated in breast cancer. Oncogene 28: 195-208; 2009.

11. Yu J, Fang Q and Meng S: Knockdown of Long noncoding RNA ENST457720 inhibits proliferation of non-small cell lung cancer cells in vitro and in vivo. Oncol Res 27: 47-53, 2018.
12. Du Y, Hao X and Liu X: Low expression of long noncoding RNA CDKN2B-AS1 in patients with idiopathic pulmonary fibrosis predicts lung cancer by regulating the p53-signaling pathway. Oncol Lett 15: 4912-4918, 2018.

13. Livak KJ and Schmittgen TD: Analysis of relative gene expression data using real-time quantitative PCR and the 2(-Delta Delta C(T)) method. Methods 25: 402-408, 2001.

14. Siegel RL, Miller KD and Jemal A: Cancer statistics, 2015. CA Cancer J Clin 65: 5-29, 2015.

15. Ma YM, Peng YM, Zhu QH, Gao AH, Chao B, He QJ, Li J, $\mathrm{Hu}$ YH and Zhou YB: Novel CHOP activator LGH00168 induces necroptosis in A549 human lung cancer cells via ROS-mediated ER stress and NF-кB inhibition. Acta Pharmacol Sin 37: 1381-1390, 2016.

16. Song H, Sun W, Ye G, Ding X, Liu Z, Zhang S, Xia T, Xiao B, $\mathrm{Xi} \mathrm{Y}$ and Guo J: Long non-coding RNA expression profile in human gastric cancer and its clinical significances. J Transl Med 11: 225, 2013

17. Liu Z, Shao Y, Tan L, Shi H, Chen S and Guo J: Clinical significance of the low expression of FER1L4 in gastric cancer patients. Tumour Biol 35: 9613-9617, 2014.

18. Yue B, Sun B, Liu C, Zhao S, Zhang D, Yu F and Yan D: Long non-coding RNA Fer-1-like protein 4 suppresses oncogenesis and exhibits prognostic value by associating with miR-106a-5p in colon cancer. Cancer Sci 106: 1323-1332, 2015.

19. Ling YH, Quan Q, Xiang H, Zhu L, Chu MX, Zhang XR and Han CY: Expression profiles of differentially expressed genes affecting fecundity in goat ovarian tissues. Genet Mol Res 14: 18743-18752, 2015.

20. Wu J, Huang J, Wang W, Xu J, Yin M, Cheng N and Yin J: Long non-coding RNA Fer-1-like protein 4 acts as a tumor suppressor via miR-106a-5p and predicts good prognosis in hepatocellular carcinoma. Cancer Biomark 20: 55-65, 2017.

21. Ding F, Tang H, Nie D and Xia L: Long non-coding RNA Fer-1-like family member 4 is overexpressed in human glioblastoma and regulates the tumorigenicity of glioma cells. Oncol Lett 14: 2379-2384, 2017.

22. Bakin AV, Tomlinson AK, Bhowmick NA, Moses HL and Arteaga CL: Phosphatidylinositol 3-kinase function is required for transforming growth factor beta-mediated epithelial to mesenchymal transition and cell migration. J Biol Chem 275: 36803-36810, 2000

23. Porta C, Paglino C and Mosca A: Targeting PI3K/Akt/mTOR Signaling in Cancer. Front Oncol 4: 64, 2014.

24. Dong J, Zhai B, Sun W, Hu F, Cheng $\mathrm{H}$ and $\mathrm{Xu}$ J: Activation of phosphatidylinositol 3-kinase/AKT/snail signaling pathway contributes to epithelial-mesenchymal transition-induced multi-drug resistance to sorafenib in hepatocellular carcinoma cells. PLoS One 12: e185088, 2017.

25. Carracedo A and Pandolfi PP: The PTEN-PI3K pathway: Of feedbacks and cross-talks. Oncogene 27: 5527-5541, 2008.

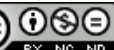

This work is licensed under a Creative Commons Attribution-NonCommercial-NoDerivatives 4.0 International (CC BY-NC-ND 4.0) License. 